\title{
Broken Charges Associated with Classical Spacetime Symmetries under Canonical Transformation in Real Scalar Field Theory
}

\author{
Susobhan Mandal \\ Department of Physical Sciences, Indian Institute of Science Education and Research Kolkata, Mohanpur, India \\ Email: sm17rs045@iiserkol.ac.in
}

How to cite this paper: Mandal, S. (2019) Broken Charges Associated with Classical Spacetime Symmetries under Canonical Transformation in Real Scalar Field Theory. Journal of High Energy Physics, Gravitation and Cosmology, 5, 167-180.

https://doi.org/10.4236/jhepgc.2019.51009

Received: November 5, 2018

Accepted: December 17, 2018

Published: December 20, 2018

Copyright $\odot 2019$ by author and Scientific Research Publishing Inc. This work is licensed under the Creative Commons Attribution International License (CC BY 4.0).

http://creativecommons.org/licenses/by/4.0/

\begin{abstract}
We know from Noether's theorem that there is a conserved charge for every continuous symmetry. In General Relativity, Killing vectors describe the spacetime symmetries and to each such Killing vector field, we can associate conserved charge through stress-energy tensor of matter which is mentioned in the article. In this article, I show that under simple set of canonical transformation of most general class of Bogoliubov transformation between creation, annihilation operators, those charges associated with spacetime symmetries are broken. To do that, I look at stress-energy tensor of real scalar field theory (as an example) in curved spacetime and show how it changes under simple canonical transformation which is enough to justify our claim. Since doing Bogoliubov transformation is equivalent to coordinate transformation which according to Einstein's equivalence principle is equivalent to turn on effect of gravity, therefore, we can say that under the effect of gravity those charges are broken.
\end{abstract}

\section{Keywords}

Noether's Theorem, Killing Vector Field, Einstein's Equivalence, Broken Charges

\section{Introduction}

In [1], I have shown that internal symmetry like U(1) symmetry of matter field under canonical transformation [2] [3] breaks down. We all know that doing Bogoliubov transformation [4] is equivalent to turning on the effect of gravity by going into different frame. And Killing vector fields [5] [6] of a spacetime decides the symmetries of that spacetime. We also know that stress-energy tensor [5] [6] of matter fields in curved spacetime is covariantly conserved [6]. 
Therefore, let $T^{\mu \nu}(x)$ be the stress-energy tensor of the matter field and $\xi^{\mu}$ be a killing vector field of a spacetime we consider then one can show that $T^{\mu v} \xi_{v}$ is a covariantly conserved current [6]

$$
\nabla_{\mu}\left(T^{\mu v} \xi_{v}\right)=\left(\nabla_{\mu} T^{\mu v}\right) \xi_{v}+\frac{1}{2} T^{\mu v}\left(\nabla_{\mu} \xi_{v}+\nabla_{v} \xi_{\mu}\right)=0
$$

where we have used the symmetry property of stress-energy tensor under contravariant indices, Killing equation [5] [6] [7] and covariantly conserved property of stress-energy tensor. Therefore, we can say that from stress-energy tensor we can construct the generators of spacetime symmetry transformation which is $\mathcal{Q}=\int \sqrt{-g(x)} \mathrm{d}^{3} x T^{0 v}(x) \xi_{v}(x)$ defined on spacelike hypersurfaces. In this article, I will show that under Bogoliubov transformation, generator (conserved charge) $\mathcal{Q}=\int \sqrt{-g(x)} \mathrm{d}^{3} x T^{0 v}(x) \xi_{v}(x)$ is broken [8] [9].

Here also I use the concept that in thermodynamic limit or infinite volume limit Bogoliubov tranformation creates two inequivalent representations of two disjoint Fock space which is often used in quantum many body systems. Because of such inequivalent disjoint vector spaces, the operators both in original form and ones after transformation have their own separate domain to act on states.

\section{Breakdown of Translation Invariance of Minkowski Spacetime under Canonical Transformation}

Here I am going to discuss my main motivation behind this work. Let's consider a massive real scalar field theory in Minkowski spacetime with field decomposition

$$
\begin{aligned}
& \hat{\phi}(x) \\
& =\int \frac{\mathrm{d}^{3} k}{\sqrt{(2 \pi)^{3} 2 \omega_{k}}}\left[\hat{a}_{k} \mathrm{e}^{-i k \cdot x}+\hat{a}_{k}^{\dagger} \mathrm{e}^{i k \cdot x}\right]
\end{aligned}
$$

therefore $\hat{\phi}(x) \rightarrow \hat{\phi}(x-a)$ is equivalent to doing following transformation

$$
\begin{aligned}
& \hat{a}_{k} \rightarrow \mathrm{e}^{i k \cdot a} \hat{a}_{k} \\
& \hat{a}_{k}^{\dagger} \rightarrow \mathrm{e}^{-i k \cdot a} \hat{a}_{k}^{\dagger}
\end{aligned}
$$

The Hamiltonian of the matter field becomes

$$
\hat{H}=\int_{k} \varepsilon_{k} \hat{a}_{k}^{\dagger} \hat{a}_{k}
$$

which is invariant under following transformation

$$
\begin{aligned}
& \hat{a}_{k} \rightarrow \mathrm{e}^{i k \cdot a} \hat{a}_{k} \\
& \hat{a}_{k}^{\dagger} \rightarrow \mathrm{e}^{-i k \cdot a} \hat{a}_{k}^{\dagger}
\end{aligned}
$$

where $\boldsymbol{a}$ is a 4-vector, which we would expect because spacetime translation is Killing vector fields in Minkowski spacetime.

Under the canonical transformation mentioned in [1]

$$
\hat{c}_{\boldsymbol{k}}(\theta)=\cosh \theta_{\boldsymbol{k}} \hat{a}_{\boldsymbol{k}}-\sinh \theta_{\boldsymbol{k}} \hat{a}_{-\boldsymbol{k}}^{\dagger}
$$

the above Hamiltonian becomes 


$$
\begin{aligned}
\hat{H} & =\int \mathrm{d}^{3} k \varepsilon_{k} \hat{a}_{k}^{\dagger} \hat{a}_{k} \\
& =\int \mathrm{d}^{3} k \varepsilon_{\vec{k}}\left[\cosh \theta_{k} \hat{c}_{k}^{\dagger}+\sinh \theta_{k} \hat{c}_{-k}\right]\left[\cosh \theta_{k} \hat{c}_{k}+\sinh \theta_{k} \hat{c}_{-k}^{\dagger}\right] \\
& =\int \mathrm{d}^{3} k \varepsilon_{k}\left[\cosh ^{2} \theta_{k} \hat{c}_{k}^{\dagger} \hat{c}_{k}+\sinh ^{2} \theta_{k} \hat{c}_{-k} \hat{c}_{-k}^{\dagger}+\sinh \theta_{k} \cosh \theta_{k}\left(\hat{c}_{-k} \hat{c}_{k}+\hat{c}_{k}^{\dagger} \hat{c}_{-k}^{\dagger}\right)\right] \\
& \simeq \int \mathrm{d}^{3} k \varepsilon_{k}\left[\left(\cosh ^{2} \theta_{k}+\sinh ^{2} \theta_{-k}\right) \hat{c}_{k}^{\dagger} \hat{c}_{k}+\sinh \theta_{k} \cosh \theta_{k}\left(\hat{c}_{-k} \hat{c}_{k}+\hat{c}_{k}^{\dagger} \hat{c}_{-k}^{\dagger}\right)\right]
\end{aligned}
$$

where we have used the inverse transformation

$$
\begin{aligned}
& \hat{c}_{k}(\theta)=\cosh \theta_{k} \hat{a}_{k}-\sinh \theta_{k} \hat{a}_{-k}^{\dagger} \\
& \hat{c}_{k}^{\dagger}(\theta)=\cosh \theta_{k} \hat{a}_{k}^{\dagger}-\sinh \theta_{k} \hat{a}_{-k} \\
& \hat{a}_{k}=\cosh \theta_{k} \hat{c}_{k}+\sinh \theta_{k} \hat{c}_{-k}^{\dagger} \\
& \hat{a}_{k}^{\dagger}=\cosh \theta_{k} \hat{c}_{k}^{\dagger}+\sinh \theta_{k} \hat{c}_{-k}
\end{aligned}
$$

Note that because of the last term in the transformed Hamiltonian it breaks the invariance under the tranformation

$$
\begin{aligned}
& \hat{c}_{\boldsymbol{k}} \rightarrow \mathrm{e}^{i k \cdot a} \hat{c}_{\boldsymbol{k}} \\
& \hat{c}_{\boldsymbol{k}}^{\dagger} \rightarrow \mathrm{e}^{-i k \cdot a} \hat{c}_{\boldsymbol{k}}^{\dagger}
\end{aligned}
$$

If one notes carefully he/she would find that for this case only the time translational symmetry breaks but the spatial translation symmetry is maintained. But this is just an example. If we rather take more general transformation

$$
\hat{a}_{\boldsymbol{k}}=\int \mathrm{d}^{3} k^{\prime}\left[\alpha\left(\boldsymbol{k}^{\prime}, \boldsymbol{k}\right) \hat{c}_{\boldsymbol{k}^{\prime}}+\beta\left(\boldsymbol{k}^{\prime}, \boldsymbol{k}\right) \hat{c}_{\boldsymbol{k}^{\prime}}^{\dagger}\right]
$$

such that

$$
\int \mathrm{d}^{3} k^{\prime}\left[\left|\alpha\left(\boldsymbol{k}^{\prime}, \boldsymbol{k}\right)\right|^{2}-\left|\beta\left(\boldsymbol{k}^{\prime}, \boldsymbol{k}\right)\right|^{2}\right]=1, \forall \boldsymbol{k}
$$

Then Hamiltonian becomes highly non-local

$$
\begin{aligned}
\hat{H}= & \int \mathrm{d}^{3} k_{1} \mathrm{~d}^{3} k_{2} \mathrm{~d}^{3} k\left[\varepsilon_{\boldsymbol{k}}\left[\alpha^{*}\left(\boldsymbol{k}_{1}, \boldsymbol{k}\right) \hat{c}_{\boldsymbol{k}_{1}}^{\dagger}+\beta^{*}\left(\boldsymbol{k}_{1}, \boldsymbol{k}\right) \hat{c}_{\boldsymbol{k}_{1}}\right]\right. \\
& \left.\times\left[\alpha\left(\boldsymbol{k}_{2}, \boldsymbol{k}\right) \hat{c}_{\boldsymbol{k}_{2}}+\beta\left(\boldsymbol{k}_{2}, \boldsymbol{k}\right) \hat{c}_{\boldsymbol{k}_{2}}^{\dagger}\right]\right] \\
= & \int \mathrm{d}^{3} k_{1} \mathrm{~d}^{3} k_{2} \mathrm{~d}^{3} k \varepsilon_{\boldsymbol{k}}\left[\alpha^{*}\left(\boldsymbol{k}_{1}, \boldsymbol{k}\right) \alpha\left(\boldsymbol{k}_{2}, \boldsymbol{k}\right) \hat{c}_{\boldsymbol{k}_{1}}^{\dagger} \hat{c}_{\boldsymbol{k}_{2}}\right. \\
& +\beta^{*}\left(\boldsymbol{k}_{1}, \boldsymbol{k}\right) \beta\left(\boldsymbol{k}_{2}, \boldsymbol{k}\right) \hat{c}_{\boldsymbol{k}_{1}} \hat{c}_{\boldsymbol{k}_{2}}^{\dagger}+\beta^{*}\left(\boldsymbol{k}_{1}, \boldsymbol{k}\right) \alpha\left(\boldsymbol{k}_{2}, \boldsymbol{k}\right) \hat{c}_{\boldsymbol{k}_{1}} \hat{c}_{\boldsymbol{k}_{2}} \\
& \left.+\alpha^{*}\left(\boldsymbol{k}_{1}, \boldsymbol{k}\right) \beta\left(\boldsymbol{k}_{2}, \boldsymbol{k}\right) \hat{c}_{\boldsymbol{k}_{1}}^{\dagger} \hat{c}_{\boldsymbol{k}_{2}}^{\dagger}\right]
\end{aligned}
$$

This expression undoubtedly suggests that the above non-local Hamiltonian is not invariant under the tranformation

$$
\begin{aligned}
& \hat{c}_{\boldsymbol{k}} \rightarrow \mathrm{e}^{i k \cdot a} \hat{c}_{\boldsymbol{k}} \\
& \hat{c}_{\boldsymbol{k}}^{\dagger} \rightarrow \mathrm{e}^{-i k \cdot a} \hat{c}_{\boldsymbol{k}}^{\dagger}
\end{aligned}
$$

which shows that under most general Bogoliubov transformation spacetime translation symmetry of Minkowski spacetime breaks down.

As an example we want to mention cosmological particle creation example shown in [10] where these Bogoliubov coeffecients are calculated for conformal spacetime with following metric 


$$
\begin{aligned}
& \mathrm{d} s^{2}=a^{2}(\eta)\left(\mathrm{d} \eta^{2}-\mathrm{d} x^{2}-\mathrm{d} y^{2}-\mathrm{d} z^{2}\right) \\
& a^{2}(\eta)=A+B \tanh \rho \eta
\end{aligned}
$$

where $A, B, \rho$ are some constant parameters. Note that this spacetime is flat in asymptotic times $\eta \rightarrow \pm \infty$. In this case they found

$$
\begin{aligned}
& \cosh \theta_{\boldsymbol{k}}=\frac{\sinh ^{2}\left(\frac{\pi \omega_{+}}{\rho}\right)}{\sinh \left(\frac{\pi \omega_{\text {in }}}{\rho}\right) \sinh \left(\frac{\pi \omega_{\text {out }}}{\rho}\right)} \\
& \sinh \theta_{\boldsymbol{k}}=\frac{\sinh ^{2}\left(\frac{\pi \omega_{-}}{\rho}\right)}{\sinh \left(\frac{\pi \omega_{\text {in }}}{\rho}\right) \sinh \left(\frac{\pi \omega_{\text {out }}}{\rho}\right)} \\
& \omega_{ \pm}(\boldsymbol{k})=\frac{1}{2}\left(\omega_{\text {out }} \pm \omega_{\text {in }}\right) \\
& \omega_{\text {out }}=\sqrt{\boldsymbol{k}^{2}+m^{2}(A+B)} \\
& \omega_{\text {in }}=\sqrt{\boldsymbol{k}^{2}+m^{2}(A-B)}
\end{aligned}
$$

Note that to go from one asymptotic region to another asymptotic region we have to do basically time translation and corresponding Bogoliubov transformation is defined by above coeffecients. According to our theory this example breaks time translational symmetry in the transformed Hamiltonian but in asymptotic time region metric is time translational invariant.

So, through the generalization of this mathematical idea we want to show that if there are 2 observers living in 2 different spacetime which are connected through some coordinate transformation then one observer can know about a matter field living in other observer's spacetime through Bogoliubov transformation but through that process for him charges defined with the help of stressenergy tensor associated with Killing vector fields of other spacetime are broken. This is our claim which we want to show.

\section{Mathematical Framework of Scalar Field in Curved Spactime}

\subsection{The Quantized Real Scalar Field}

Let's consider a real scalar field theory in some general curved spacetime described following action

$$
S=-\frac{1}{2} \int \mathrm{d}^{4} x \sqrt{-g(x)}\left[g^{\mu \nu}(x) \partial_{\mu} \phi(x) \partial_{\nu} \phi(x)+m^{2} \phi^{2}(x)\right]
$$

The Euler-Lagrange equation will take following form [1] [11]

$$
\left[-\square+m^{2}\right] \phi(x)=0, \square \phi=\frac{1}{\sqrt{-g}} \partial_{\lambda}\left[\sqrt{-g} g^{\lambda v} \partial_{\nu} \phi\right]
$$

For Canonical quantization we need the conjugate momentum corresponding to the field $\phi(x)$ 


$$
\Pi=\frac{\partial \mathcal{L}}{\partial \partial_{0} \phi}=-\sqrt{-g} \partial^{0} \phi
$$

Using this information one can easily write down the Hamiltonian density

$$
\mathcal{H}=\Pi \partial_{0} \phi-\mathcal{L}=\frac{1}{2} \sqrt{-g}\left[-g^{00}\left(\partial_{0} \phi\right)^{2}+g^{i j} \partial_{i} \phi \partial_{j} \phi+m^{2} \phi^{2}\right]
$$

and in other way we can write the Hamiltonian as

$$
H=\int \mathrm{d}^{3} x \mathcal{H}=\frac{1}{2} \int \mathrm{d}^{3} x \Pi \overrightarrow{\partial_{0}} \phi=\frac{1}{2} \int \mathrm{d}^{3} x\left[\Pi \partial_{0} \phi-\partial_{0} \Pi \phi\right]
$$

which can be shown through little bit of algebra.

Now note that the last formula give us

$$
[\hat{\phi}(t, \boldsymbol{x}), \hat{H}(t)]=i \partial_{0} \hat{\phi}(t, \boldsymbol{x})
$$

which we get through the quantization prescription

$$
\begin{aligned}
& {\left[\hat{\phi}(t, \boldsymbol{x}), \hat{\Pi}\left(t, \boldsymbol{x}^{\prime}\right)\right]=i \delta^{(3)}\left(\boldsymbol{x}-\boldsymbol{x}^{\prime}\right)} \\
& {\left[\hat{\phi}(t, \boldsymbol{x}), \hat{\phi}\left(t, \boldsymbol{x}^{\prime}\right)\right]=0=\left[\hat{\Pi}(t, \boldsymbol{x}), \hat{\Pi}\left(t, \boldsymbol{x}^{\prime}\right)\right]}
\end{aligned}
$$

\subsection{Stress-Energy Tensor}

Einstein-Hilbert action is following

$$
S=\int \frac{\mathrm{d}^{4} x}{16 \pi} \sqrt{-g(x)} R(x)+S_{\text {matter }}
$$

with Einstein-equation as the Euler-Lagrange equation

$$
G_{\alpha \beta}=R_{\alpha \beta}-\frac{1}{2} g_{\alpha \beta} R=8 \pi T_{\alpha \beta}
$$

where $R_{\alpha \beta}$ is Ricci tensor and $R$ is Ricci scalar. And the stress-energy tensor $T^{\alpha \beta}$ is given by

$$
T^{\alpha \beta}(x)=-\frac{2}{\sqrt{-g}} \frac{\delta S_{\text {matter }}}{\delta g_{\alpha \beta}}
$$

which in our case becomes

$$
T_{\mu \nu}=\partial_{\mu} \phi \partial_{\nu} \phi-\frac{1}{2} g_{\mu \nu}(\partial \phi)^{2}-\frac{1}{2} g_{\mu \nu} m^{2} \phi^{2}
$$

Now note that conservation of stress-energy tensor comes from the fact that matter action is invariant under spacetime diffeomorphisms

$$
\begin{aligned}
\delta S & =0=\int \frac{\delta S_{\text {matter }}}{\delta g_{\alpha \beta}(x)} \delta g_{\alpha \beta}(x) \mathrm{d}^{4} x \\
& =-\frac{1}{2} \int \sqrt{-g} T^{\alpha \beta}\left(\xi_{\alpha ; \beta}+\xi_{\beta ; \alpha}\right) \mathrm{d}^{4} x=-\int \sqrt{-g} T^{\alpha \beta} \xi_{\beta ; \alpha} \\
& =-\underbrace{\int \nabla_{\alpha}\left[\sqrt{-g} T^{\alpha \beta} \xi_{\beta}\right]}_{\text {surfaceterm }}+\int \sqrt{-g} \nabla_{\alpha} T^{\alpha \beta} \xi_{\beta} \\
& =\int \sqrt{-g} \nabla_{\alpha} T^{\alpha \beta} \xi_{\beta} \\
\Rightarrow & \nabla_{\alpha} T^{\alpha \beta}=0
\end{aligned}
$$


where $\xi_{\alpha}$ is a Killing vector field of the spacetime.

\subsection{Fock Space}

Let us consider a complete set of mode solutions $\left\{f_{j}, f_{j}^{*}\right\}$ of the Klein-Gordon equation, with $\{j\}$ being a set of labels which distinguish independent solutions. The modes are normalised with respect to the inner product defined in following way

$$
\langle f, g\rangle=-i \int_{V} \sqrt{-g} \mathrm{~d}^{3} x\left[f^{*}(t, x) \overrightarrow{\partial^{0}} g(t, x)\right]
$$

such that solutions are orthonormalized in following manner

$$
\begin{aligned}
& \left\langle f_{j}, f_{j^{\prime}}\right\rangle=\delta_{i j j^{\prime}},\left\langle f_{j}^{*}, f_{j^{\prime}}^{*}\right\rangle=-\delta_{j j^{\prime}} \\
& \left\langle f_{j}^{*}, f_{j^{\prime}}\right\rangle=0=\left\langle f_{j}, f_{j^{\prime}}^{*}\right\rangle
\end{aligned}
$$

And one can also show that this definition of inner product is time-independent [12].

Assuming that the inner product is well defined, the corresponding completeness relation can be written as:

$$
\sum_{j}\left[f_{j}(t, \boldsymbol{x}) \partial^{0} f_{j}^{*}\left(t, \boldsymbol{x}^{\prime}\right)-\partial^{0} f_{j}(t, \boldsymbol{x}) f_{j}^{*}\left(t, \boldsymbol{x}^{\prime}\right)\right]=-\frac{i}{\sqrt{-g}} \delta^{(3)}\left(\boldsymbol{x}-\boldsymbol{x}^{\prime}\right)
$$

Now we write field operator in terms of mode decomposition

$$
\hat{\phi}(x)=\sum_{j}\left[\hat{a}_{j} f_{j}(x)+\hat{a}_{j}^{\dagger} f_{j}^{*}(x)\right]
$$

Second quantisation promotes $\hat{\phi}(x)$ to an operator, called the field operator, obeying the commutation relation mentioned earlier. Consequently, the coefficients $\hat{a}_{j}$ and $\hat{a}_{j}^{\dagger}$ obey the following algebra:

$$
\begin{aligned}
& {\left[\hat{a}_{j}, \hat{a}_{j^{\prime}}^{\dagger}\right]=\delta_{j j^{\prime}}} \\
& {\left[\hat{a}_{j}, \hat{a}_{j^{\prime}}\right]=0=\left[\hat{a}_{j}^{\dagger}, \hat{a}_{j^{\prime}}^{\dagger}\right]}
\end{aligned}
$$

And vacuum state $|0\rangle$ is defined such that

$$
\hat{a}_{j}|0\rangle=0, \forall j
$$

Applying products of creation operators $\hat{a}_{j}^{\dagger}$ to the vacuum state creates multiparticle states, which form a basis of the Fock space

$$
\left|j_{1} j_{2} \cdots j_{n}\right\rangle=\frac{1}{(n !)^{\frac{3}{2}}} \sum_{\sigma \in S_{n}} \prod_{i=1}^{n} \hat{a}_{\sigma_{\sigma_{i}}}^{\dagger}|0\rangle
$$

The identity operator can be written in terms of the basis vectors

$$
\mathbb{I}=|0\rangle\left\langle 0\left|+\sum_{n=1}^{\infty} \sum_{j_{1}} \cdots \sum_{j_{n}}\right| j_{1} j_{2} \cdots j_{n}\right\rangle\left\langle j_{1} j_{2} \cdots j_{n}\right|
$$

In terms of one-particle operators, the stress-energy tensor can be written in following way 


$$
\begin{aligned}
& \hat{T}_{\mu \nu}=\sum_{j, k}\left[\hat{a}_{j} \hat{a}_{k} \mathcal{T}_{\mu \nu}\left(f_{j}, f_{k}\right)+\hat{a}_{j}^{\dagger} \hat{a}_{k}^{\dagger} \mathcal{T}_{\mu \nu}\left(f_{j}^{*}, f_{k}^{*}\right)\right. \\
& \left.\quad+\hat{a}_{j}^{\dagger} \hat{a}_{k} \mathcal{T}_{\mu \nu}\left(f_{j}^{*}, f_{k}\right)+\hat{a}_{j} \hat{a}_{k}^{\dagger} \mathcal{T}_{\mu \nu}\left(f_{j}, f_{k}^{*}\right)\right] \\
& \mathcal{T}_{\mu \nu}\left(f_{j}, f_{k}\right)=\nabla_{\mu} f_{j} \nabla_{v} f_{k}-\frac{1}{2} g_{\mu \nu}\left(g^{\alpha \beta} \nabla_{\alpha} f_{j} \nabla_{\beta} f_{k}\right)-\frac{1}{2} g_{\mu \nu} m^{2} f_{j} f_{k}
\end{aligned}
$$

Looking at the first 2 terms in stress-energy tensor operator one might think it already breaks $U(1)$ symmetry but it actually not. To know that we have to go further.

Let us specialise further to mode solutions of the Klein-Gordon equation which satisfy the eigenvalue equations

$$
\begin{aligned}
& i \partial_{t} f_{j}=\tilde{\omega}_{j} f_{j}, \quad i \partial_{t} f_{j}^{*}=-\tilde{\omega}_{j} f_{j}^{*} \\
& -i \partial^{t} f_{j}=\omega_{j} f_{j}, \quad-i \partial^{t} f_{j}^{*}=-\omega_{j} f_{j}^{*}
\end{aligned}
$$

An integration of these equations shows that $f_{j} \simeq \mathrm{e}^{i \tilde{\omega}_{j} t}$. Hence, $\tilde{\omega}_{j}$ can be interpreted as the frequency of the mode $j$. Under the assumptions, the normalisation conditions and the completeness relation take the form:

$$
\begin{aligned}
& \left(\omega_{j}+\omega_{j^{\prime}}\right) \int \sqrt{-g} \mathrm{~d}^{3} x f_{j}^{*}(t, \boldsymbol{x}) f_{j^{\prime}}(t, \boldsymbol{x})=\delta_{i j^{\prime}} \\
& \left(\omega_{j}-\omega_{j^{\prime}}\right) \int \sqrt{-g} \mathrm{~d}^{3} x f_{j}(t, \boldsymbol{x}) f_{j^{\prime}}(t, \boldsymbol{x})=0 \\
& \sum_{j} \omega_{j}\left[f_{j}^{*}(t, \boldsymbol{x}) f_{j^{\prime}}(t, \boldsymbol{x})+f_{j}(t, \boldsymbol{x}) f_{j^{\prime}}^{*}(t, \boldsymbol{x})\right]=\frac{1}{\sqrt{-g}} \delta^{(3)}\left(\boldsymbol{x}-\boldsymbol{x}^{\prime}\right)
\end{aligned}
$$

which requires $\omega_{j} \geq 0 \quad$ (i.e., instead of the eigenvalue $\tilde{\omega}_{j}$ of the Hamiltonian). Thus, the norm and frequency of a mode $f_{j}$ can have opposite signs if $\omega_{j} \tilde{\omega}_{j}<0$, forcing modes with negative frequency in the set of particle modes which we don't want.

Using the definition of conjugate momentum and Hamiltonian one can derive [13]

$$
\hat{H}=\frac{1}{2} \sum_{j} \tilde{\omega}_{j}\left[\hat{a}_{j}^{\dagger} \hat{a}_{j}+\hat{a}_{j} \hat{a}_{j}^{\dagger}\right]=\sum_{j} \tilde{\omega}_{j}\left[\hat{a}_{j}^{\dagger} \hat{a}_{j}+\frac{1}{2}\right]
$$

Above equation shows that particles for which $\tilde{\omega}_{j}<0$ make negative contributions to the total Hamiltonian of the system, while particles for which $\tilde{\omega}_{j}$ vanishes do not contribute.

\subsection{Finite Temperature Field Theory}

The concept of temperature is implemented by considering a quantum state containing a thermal distribution of particle states, with the Hamiltonian operator $\hat{H}$ playing the role of energy. The expectation value of an operator $\hat{A}$ in a thermal state at a finite inverse temperature $\beta=T^{-1}$ is defined as

$$
\begin{aligned}
& \langle\hat{A}\rangle_{\beta}=\frac{1}{\mathcal{Z}} \operatorname{Tr}\left[\mathrm{e}^{-\beta \hat{H}} \hat{A}\right]=\frac{1}{\mathcal{Z}} \sum_{n=0}^{\infty}\left[\sum_{j_{1}, \cdots, j_{n}}\left\langle j_{1} \cdots j_{n}\left|\mathrm{e}^{-\beta \hat{H}}\right| j_{1} \cdots j_{n}\right\rangle\right] \\
& \mathcal{Z}=\operatorname{Tre}^{-\beta \hat{H}}
\end{aligned}
$$

It is now hard to show that [14] [15] 


$$
\left\langle\hat{a}_{j}^{\dagger} \hat{a}_{j^{\prime}}\right\rangle_{\beta}=\frac{\delta_{i j^{\prime}}}{\mathrm{e}^{\beta \tilde{\omega}_{j}}-1},\left\langle\hat{a}_{j} \hat{a}_{j^{\prime}}^{\dagger}\right\rangle_{\beta}=\frac{\delta_{j j^{\prime}}}{1-\mathrm{e}^{-\beta \tilde{\omega}_{j}}}, \quad\left\langle\hat{a}_{j} \hat{a}_{j^{\prime}}\right\rangle_{\beta}=0=\left\langle\hat{a}_{j}^{\dagger} \hat{a}_{j^{\prime}}^{\dagger}\right\rangle_{\beta}
$$

Notice because of $U(1)$ invariance of the Hamiltonian, in thermal expectation values of stress-energy tensor first 2 terms won't contribute and therefore we will get

$$
\left\langle: \hat{T}_{\mu \nu}:\right\rangle_{\beta}=\sum_{j} \frac{1}{\mathrm{e}^{\beta \tilde{\omega}_{j}}-1}\left[\mathcal{T}_{\mu \nu}\left(f_{j}^{*}, f_{j^{\prime}}\right)+\mathcal{T}_{\mu \nu}\left(f_{j}, f_{j^{\prime}}^{*}\right)\right]
$$

but those term will contribute when we are considering $\left\langle: \hat{T}_{\mu \nu}(x) \hat{T}_{\alpha \beta}(y):\right\rangle_{\beta}$. Just because such 2 terms are present in stress-energy operator does not implies $U(1)$ symmetry breaking but if it presents in generator of certain transformation then only we say $U(1)$ symmetry is breaking. Since generator of time-translation is $\hat{T}^{00}$ which is same as hamiltonian operator $\hat{H}$ therefore we are sure that $U(1)$ symmetry is not broken therefore charge is conserved. On the other hand if we find $\hat{T}^{0 i}$ does not contain those 2 terms then charge associated with it does not commute with generators of $\mathrm{U}(1)$ action.

\section{Canonical Transformation}

Under following canonical canonical transformation

$$
\begin{aligned}
& \hat{a}_{j}=\cosh \theta_{j} \hat{c}_{j}+\sinh \theta_{j} \hat{c}_{j}^{\dagger} \\
& \hat{a}_{j}^{\dagger}=\cosh \theta_{j} \hat{c}_{j}^{\dagger}+\sinh \theta_{j} \hat{c}_{j}
\end{aligned}
$$

Then in similar way as in [1] where we use finite temperature field theory concepts from [16] [17] [18] [19] we can write partition function under above transformation in following path integral manner

$$
\begin{aligned}
\mathcal{Z}=\int \mathcal{D} \bar{\phi} \mathcal{D} \phi \mathrm{e}^{S_{E}(\bar{\phi}, \phi)} & \\
S_{E}(\bar{\phi}, \phi)= & \frac{1}{\hbar} \int_{0}^{\beta \hbar} \mathrm{d} \tau \sum_{j}\left[\bar{\phi}_{j}(\tau)\left(\hbar \partial_{\tau}-\left(\cosh ^{2} \theta_{j}+\sinh ^{2} \theta_{j}\right) \xi_{j}\right) \phi_{j}(\tau)\right. \\
& \left.-\xi_{j} \sinh \theta_{j} \cosh \theta_{j}\left(\phi_{j}(\tau) \phi_{j}(\tau)+\bar{\phi}_{j}(\tau) \bar{\phi}_{j}(\tau)\right)\right]
\end{aligned}
$$

which can be written in matrix representation in following way (denoting $\chi_{j}=\cosh ^{2} \theta_{j}+\sinh ^{2} \theta_{j}, \eta_{j}=\cosh \theta_{j} \sinh \theta_{j}, \quad \xi_{j}=\tilde{\omega}_{j}-\mu$ where $\mu$ is the chemical potential)

$$
\begin{aligned}
& S_{E}(\bar{\phi}, \phi)=\frac{1}{\hbar} \int_{0}^{\beta \hbar} \mathrm{d} \tau \sum_{j}\left(\bar{\phi}_{j}(\tau) \quad \phi_{j}(\tau)\right) \\
& \times\left[\begin{array}{cc}
\frac{1}{2}\left(\hbar \partial_{\tau}-\xi_{j} \chi_{j}\right) & \xi_{j} \eta_{j} \\
\xi_{j} \eta_{j} & \frac{1}{2}\left(\hbar \bar{\partial}_{\tau}-\xi_{j} \chi_{j}\right)
\end{array}\right] \times\left(\begin{array}{c}
\phi_{j}(\tau) \\
\bar{\phi}_{j}(\tau)
\end{array}\right) \\
& =\beta \sum_{n} \int \mathrm{d}^{3} k\left(\bar{\phi}_{j}\left(\omega_{n}\right) \quad \phi_{j}\left(-\omega_{n}\right)\right) \\
& \times\left[\begin{array}{cc}
\frac{1}{2}\left(i \hbar \omega_{n}-\xi_{j} \chi_{j}\right) & \xi_{j} \eta_{j} \\
\xi_{j} \eta_{j} & \frac{1}{2}\left(-i \hbar \omega_{n}-\xi_{j} \chi_{j}\right)
\end{array}\right] \times\left(\begin{array}{c}
\phi_{j}\left(\omega_{n}\right) \\
\bar{\phi}_{j}\left(-\omega_{n}\right)
\end{array}\right)
\end{aligned}
$$


Now we define generating functional to get the correlation functions of any order. It is defined as(from now on we consider $\hbar=1$ )

$$
\mathcal{Z}[J, \bar{J}]=\frac{1}{\mathcal{Z}} \int \mathcal{D} \phi \mathcal{D} \bar{\phi} \mathrm{e}^{-S_{E}(\phi, \bar{\phi})} \times \mathrm{e}^{\left.\sum \sum \bar{J}_{j}\left(\omega_{n}\right) \phi_{j}\left(\omega_{n}\right)+J_{j}\left(\omega_{n}\right) \bar{\phi}_{j}\left(\omega_{n}\right)\right)}=\mathrm{e}^{\bar{J} \mathcal{G} J}
$$

where $\bar{J} \mathcal{G} J$ denotes a matrix multiplication with sum over modes and $\mathcal{G}$ is the propagator matrix or 2-point function matrix. Let's write down $\bar{J} \mathcal{G} J$ explicitly

$$
\begin{aligned}
& \bar{J} \mathcal{G} J=\sum_{n} \sum_{j}\left(\bar{J}_{j}\left(\omega_{n}\right) \quad J_{j}\left(-\omega_{n}\right)\right) \times \frac{4}{\omega_{n}^{2}+\xi_{j}^{2}\left(\chi_{j}^{2}-4 \eta_{j}^{2}\right)} \\
& \times\left[\begin{array}{cc}
\frac{1}{2}\left(-i \omega_{n}-\xi_{j} \chi_{j}\right) & -\xi_{j} \eta_{j} \\
-\xi_{j} \eta_{j} & \frac{1}{2}\left(i \omega_{n}-\xi_{j} \chi_{j}\right)
\end{array}\right]\left(\begin{array}{c}
J_{j}\left(\omega_{n}\right) \\
\bar{J}_{j}\left(-\omega_{n}\right)
\end{array}\right)
\end{aligned}
$$

Now let's do the matrix multiplication and write down the $\bar{J} \mathcal{G} J$ explicitly

$$
\begin{aligned}
\bar{J} \mathcal{G} J= & \sum_{n} \sum_{j} \frac{4}{\omega_{n}^{2}+\xi_{j}^{2}}\left[\bar{J}_{j}\left(\omega_{n}\right)\left(-i \omega_{n}-\xi_{j} \chi_{j}\right) J_{j}\left(\omega_{n}\right)\right. \\
& \left.-\xi_{j} \eta_{j}\left(\bar{J}_{j}\left(\omega_{n}\right) \bar{J}_{j}\left(-\omega_{n}\right)+J_{j}\left(\omega_{n}\right) J_{j}\left(-\omega_{n}\right)\right)\right]
\end{aligned}
$$

Note that for this case we found out that non-vanishing 2-point functions are

$$
\begin{aligned}
\left\langle\phi_{j}\left(\omega_{n}\right) \phi_{j}\left(-\omega_{n}\right)\right\rangle & =-\frac{8 \xi_{j} \eta_{j}}{\omega_{n}^{2}+\xi_{j}^{2}} \\
\left\langle\bar{\phi}_{j}\left(\omega_{n}\right) \bar{\phi}_{j}\left(-\omega_{n}\right)\right\rangle & =-\frac{8 \xi_{j} \eta_{j}}{\omega_{n}^{2}+\xi_{j}^{2}} \\
\left\langle\bar{\phi}_{j}\left(\omega_{n}\right) \phi_{j}\left(\omega_{n}\right)\right\rangle & =\frac{4\left(-i \omega_{n}-\xi_{j} \chi_{j}\right)}{\omega_{n}^{2}+\xi_{j}^{2}}
\end{aligned}
$$

From above information itself we can write

$$
\begin{aligned}
\left\langle\hat{c}_{j} \hat{c}_{j}\right\rangle_{\beta} & =\left\langle\hat{c}_{j}^{\dagger} c_{j}^{\dagger}\right\rangle_{\beta}=-\frac{1}{4 \beta} \sum_{n} \frac{8 \xi_{j} \eta_{j}}{\omega_{n}^{2}+\xi_{j}^{2}}=\frac{1}{8 \pi i} \int_{\mathcal{c}} \frac{1}{\mathrm{e}^{\beta z}-1} \frac{8 \xi_{j} \eta_{j}}{z^{2}-\xi_{j}^{2}} \\
& =\frac{\xi_{j} \eta_{j}}{\mathrm{e}^{\beta \xi_{j}}-1}-\frac{2 \xi_{j} \eta_{j}}{\mathrm{e}^{-\beta \xi_{j}}-1}=\frac{\eta_{j}\left(\mathrm{e}^{\beta \xi_{j}}+1\right)}{\mathrm{e}^{\beta \xi_{j}}-1} \\
\left\langle\hat{c}_{j}^{\dagger} \hat{c}_{j}\right\rangle_{\beta} & =-\frac{1}{4 \beta} \sum_{n} \frac{4\left(i \omega_{n}+\xi_{j} \chi_{j}\right)}{\omega_{n}^{2}+\xi_{j}^{2}}=\frac{1}{4 \pi i} \int_{\mathcal{C}} \frac{1}{\mathrm{e}^{\beta z}-1} \frac{2\left(z+\xi_{j} \chi_{j}\right)}{z^{2}-\xi_{j}^{2}} \\
& =\frac{1}{2 \xi_{j}}\left[\frac{\xi_{j}+\chi_{j} \xi_{j}}{\mathrm{e}^{\beta \xi_{j}}-1}+\frac{\mathrm{e}^{\beta \xi_{j}}\left(-\xi_{j}+\chi_{j} \xi_{j}\right)}{\mathrm{e}^{\beta \xi_{j}}-1}\right] \\
& =\frac{1}{2} \frac{\left(1+\chi_{j}\right)}{\mathrm{e}^{\beta \xi_{j}}-1}+\frac{1}{2} \frac{\mathrm{e}^{\beta \xi_{j}}\left(-1+\chi_{j}\right)}{\mathrm{e}^{\beta \xi_{j}}-1}
\end{aligned}
$$

Now we take $\beta \rightarrow \infty$ limit when only ground state contributes, therefore we get 


$$
\begin{aligned}
& \left\langle\hat{c}_{j} \hat{c}_{j}\right\rangle=\left\langle\hat{c}_{j}^{\dagger} \hat{c}_{j}^{\dagger}\right\rangle=\eta_{j}=\frac{1}{2} \sinh 2 \theta_{j} \\
& \left\langle\hat{c}_{j}^{\dagger} \hat{c}_{j}\right\rangle=\frac{1}{2}\left(\chi_{j}-1\right)=\sinh ^{2} \theta_{j} \\
& \Rightarrow\left\langle\hat{c}_{j} \hat{c}_{j}^{\dagger}\right\rangle=\sinh ^{2} \theta_{j}+1
\end{aligned}
$$

Note that above results are vacuum expectation values because at $\beta \rightarrow \infty$ limit only ground state contributes inside trace operation in partition function.

Above result clearly shows that ground state of the hamiltonian under Bogoliubov transformation is no longer a state which is annihilated by annihilation operators and also ground state does not maintain $\mathrm{U}(1)$ invariance property which we also mentioned in [1].

Now recall that before doing Bogoliubov transformation

$$
\left\langle: \hat{T}^{\mu \nu}:\right\rangle_{\beta \rightarrow \infty}=\left\langle: \hat{T}^{\mu \nu}:\right\rangle=0
$$

whereas after doing the transformation we will have following stress-energy tensor operator

$$
\begin{aligned}
: \hat{T}^{\mu v}:= & \sum_{j, k}\left[\hat{a}_{j} \hat{a}_{k} \mathcal{T}_{\mu \nu}\left(f_{j}, f_{k}\right)+\hat{a}_{j}^{\dagger} \hat{a}_{k}^{\dagger} \mathcal{T}_{\mu \nu}\left(f_{j}^{*}, f_{k}^{*}\right)\right. \\
& \left.+\hat{a}_{j}^{\dagger} \hat{a}_{k} \mathcal{T}_{\mu \nu}\left(f_{j}^{*}, f_{k}\right)+\hat{a}_{k}^{\dagger} \hat{a}_{j} \mathcal{T}_{\mu \nu}\left(f_{j}, f_{k}^{*}\right)\right] \\
= & \sum_{j, k}\left[\mathcal { T } _ { \mu \nu } ( f _ { j } , f _ { k } ) \left[\cosh \theta_{j} \cosh \theta_{k} \hat{c}_{j} \hat{c}_{k}+\sinh \theta_{j} \sinh \theta_{k} \hat{c}_{j}^{\dagger} \hat{c}_{k}^{\dagger}\right.\right. \\
& \left.+\cosh \theta_{j} \sinh \theta_{k} \hat{c}_{j} \hat{c}_{k}^{\dagger}+\sinh \theta_{j} \cosh \theta_{k} \hat{c}_{j}^{\dagger} \hat{c}_{k}\right] \\
& +\mathcal{T}_{\mu \nu}\left(f_{j}^{*}, f_{k}^{*}\right)\left[\cosh \theta_{j} \cosh \theta_{k} \hat{c}_{j}^{\dagger} \hat{c}_{k}^{\dagger}+\sinh \theta_{j} \sinh \theta_{k} \hat{c}_{j} \hat{c}_{k}\right. \\
& \left.+\cosh \theta_{j} \sinh \theta_{k} \hat{c}_{j}^{\dagger} \hat{c}_{k}+\sinh \theta_{j} \cosh \theta_{k} \hat{c}_{j} \hat{c}_{k}^{\dagger}\right] \\
& +\mathcal{T}_{\mu \nu}\left(f_{j}^{*}, f_{k}\right)\left[\cosh \theta_{j} \cosh \theta_{k} \hat{c}_{j}^{\dagger} \hat{c}_{k}+\sinh \theta_{j} \sinh \theta_{k} \hat{c}_{j} \hat{c}_{k}^{\dagger}\right. \\
& \left.+\cosh \theta_{j} \sinh \theta_{k} \hat{c}_{j}^{\dagger} \hat{c}_{k}^{\dagger}+\sinh \theta_{j} \cosh \theta_{k} \hat{c}_{j} \hat{c}_{k}\right] \\
& +\mathcal{T}_{\mu \nu}\left(f_{j}, f_{k}^{*}\right)\left[\cosh \theta_{j} \cosh \theta_{k} \hat{c}_{j} \hat{c}_{k}^{\dagger}+\sinh \theta_{j} \sinh \theta_{k} \hat{c}_{j}^{\dagger} \hat{c}_{k}\right. \\
& \left.\left.+\cosh \theta_{j} \sinh \theta_{k} \hat{c}_{j} \hat{c}_{k}+\sinh \theta_{j} \cosh \theta_{k} \hat{c}_{j}^{\dagger} \hat{c}_{k}^{\dagger}\right]\right]
\end{aligned}
$$

Now we are going to calculate $\left\langle: \hat{T}^{\mu \nu}:\right\rangle$ which w.r.t new vacuum state becomes

$$
\begin{aligned}
\left\langle: \hat{T}^{\mu v}:\right\rangle= & \sum_{j}\left[\mathcal { T } ^ { \mu \nu } ( f _ { j } , f _ { j } ) \left[\cosh \theta_{j} \cosh \theta_{j}\left\langle\hat{c}_{j} \hat{c}_{j}\right\rangle+\sinh \theta_{j} \sinh \theta_{j}\left\langle\hat{c}_{j}^{\dagger} \hat{c}_{j}^{\dagger}\right\rangle\right.\right. \\
& \left.+\cosh \theta_{j} \sinh \theta_{j}\left\langle\hat{c}_{j} \hat{c}_{j}^{\dagger}\right\rangle+\sinh \theta_{j} \cosh \theta_{j}\left\langle\hat{c}_{j}^{\dagger} \hat{c}_{j}\right\rangle\right] \\
& +\mathcal{T}^{\mu \nu}\left(f_{j}^{*}, f_{j}^{*}\right)\left[\cosh \theta_{j} \cosh \theta_{j}\left\langle\hat{c}_{j}^{\dagger} \hat{c}_{j}^{\dagger}\right\rangle+\sinh \theta_{j} \sinh \theta_{j}\left\langle\hat{c}_{j} \hat{c}_{j}\right\rangle\right. \\
& \left.+\cosh \theta_{j} \sinh \theta_{j}\left\langle\hat{c}_{j}^{\dagger} \hat{c}_{j}\right\rangle+\sinh \theta_{j} \cosh \theta_{j}\left\langle\hat{c}_{j} \hat{c}_{j}^{\dagger}\right\rangle\right] \\
& +\mathcal{T}^{\mu v}\left(f_{j}^{*}, f_{j}\right)\left[\cosh \theta_{j} \cosh \theta_{j}\left\langle\hat{c}_{j}^{\dagger} \hat{c}_{j}\right\rangle+\sinh \theta_{j} \sinh \theta_{j}\left\langle\hat{c}_{j} \hat{c}_{j}^{\dagger}\right\rangle\right. \\
& \left.+\cosh \theta_{j} \sinh \theta_{j}\left\langle\hat{c}_{j}^{\dagger} \hat{c}_{j}^{\dagger}\right\rangle+\sinh \theta_{j} \cosh \theta_{j}\left\langle\hat{c}_{j} \hat{c}_{j}\right\rangle\right] \\
& +\mathcal{T}^{\mu v}\left(f_{j}, f_{j}^{*}\right)\left[\cosh \theta_{j} \cosh \theta_{j}\left\langle\hat{c}_{j} \hat{c}_{j}^{\dagger}\right\rangle+\sinh \theta_{j} \sinh \theta_{j}\left\langle\hat{c}_{j}^{\dagger} \hat{c}_{j}\right\rangle\right. \\
& \left.\left.+\cosh \theta_{j} \sinh \theta_{j}\left\langle\hat{c}_{j} \hat{c}_{j}\right\rangle+\sinh \theta_{j} \cosh \theta_{j}\left\langle\hat{c}_{j}^{\dagger} \hat{c}_{j}^{\dagger}\right\rangle\right]\right]
\end{aligned}
$$


Note that exact answer may depend on the values of

$\mathcal{T}_{\mu \nu}\left(f_{j}, f_{j}\right), \mathcal{T}_{\mu \nu}\left(f_{j}^{*}, f_{j}\right), \mathcal{T}_{\mu \nu}\left(f_{j}, f_{j}^{*}\right), \mathcal{T}_{\mu \nu}\left(f_{j}^{*}, f_{j}^{*}\right)$ but $\left\langle\hat{c}_{j} \hat{c}_{j}\right\rangle,\left\langle\hat{c}_{j}^{\dagger} \hat{c}_{j}\right\rangle,\left\langle\hat{c}_{j} \hat{c}_{j}^{\dagger}\right\rangle,\left\langle\hat{c}_{j}^{\dagger} \hat{c}_{j}^{\dagger}\right\rangle$ are all positive definite. And also note that

$$
\begin{aligned}
\left\langle: \hat{T}_{\mu}^{\mu}:\right\rangle= & \sum_{j}\left[\mathcal { T } _ { \mu } ^ { \mu } ( f _ { j } , f _ { j } ) \left[\cosh \theta_{j} \cosh \theta_{j}\left\langle\hat{c}_{j} \hat{c}_{j}\right\rangle+\sinh \theta_{j} \sinh \theta_{j}\left\langle\hat{c}_{j}^{\dagger} \hat{c}_{j}^{\dagger}\right\rangle\right.\right. \\
& \left.+\cosh \theta_{j} \sinh \theta_{j}\left\langle\hat{c}_{j} \hat{c}_{j}^{\dagger}\right\rangle+\sinh \theta_{j} \cosh \theta_{j}\left\langle\hat{c}_{j}^{\dagger} \hat{c}_{j}\right\rangle\right] \\
& +\mathcal{T}_{\mu}^{\mu}\left(f_{j}^{*}, f_{j}^{*}\right)\left[\cosh \theta_{j} \cosh \theta_{j}\left\langle\hat{c}_{j}^{\dagger} \hat{c}_{j}^{\dagger}\right\rangle+\sinh \theta_{j} \sinh \theta_{j}\left\langle\hat{c}_{j} \hat{c}_{j}\right\rangle\right. \\
& \left.+\cosh \theta_{j} \sinh \theta_{j}\left\langle\hat{c}_{j}^{\dagger} \hat{c}_{j}\right\rangle+\sinh \theta_{j} \cosh \theta_{j}\left\langle\hat{c}_{j} \hat{c}_{j}^{\dagger}\right\rangle\right] \\
& +\mathcal{T}_{\mu}^{\mu}\left(f_{j}^{*}, f_{j}\right)\left[\cosh \theta_{j} \cosh \theta_{j}\left\langle\hat{c}_{j}^{\dagger} \hat{c}_{j}\right\rangle+\sinh \theta_{j} \sinh \theta_{j}\left\langle\hat{c}_{j} \hat{c}_{j}^{\dagger}\right\rangle\right. \\
& \left.+\cosh \theta_{j} \sinh \theta_{j}\left\langle\hat{c}_{j}^{\dagger} \hat{c}_{j}^{\dagger}\right\rangle+\sinh \theta_{j} \cosh \theta_{j}\left\langle\hat{c}_{j} \hat{c}_{j}\right\rangle\right] \\
& +\mathcal{T}_{\mu}^{\mu}\left(f_{j}, f_{j}^{*}\right)\left[\cosh \theta_{j} \cosh \theta_{j}\left\langle\hat{c}_{j} \hat{c}_{j}^{\dagger}\right\rangle+\sinh \theta_{j} \sinh \theta_{j}\left\langle\hat{c}_{j}^{\dagger} \hat{c}_{j}\right\rangle\right. \\
& \left.\left.+\cosh \theta_{j} \sinh \theta_{j}\left\langle\hat{c}_{j} \hat{c}_{j}\right\rangle+\sinh \theta_{j} \cosh \theta_{j}\left\langle\hat{c}_{j}^{\dagger} \hat{c}_{j}^{\dagger}\right\rangle\right]\right]
\end{aligned}
$$

And note that

$$
\begin{aligned}
& \mathcal{T}_{\mu}^{\mu}\left(f_{j}, f_{j}\right)=-m^{2} f_{j}^{2} \\
& \mathcal{T}_{\mu}^{\mu}\left(f_{j}^{*}, f_{j}\right)=\mathcal{T}_{\mu}^{\mu}\left(f_{j}, f_{j}^{*}\right)=-m^{2}\left|f_{j}\right|^{2} \\
& \mathcal{T}_{\mu}^{\mu}\left(f_{j}^{*}, f_{j}^{*}\right)=-m^{2} f_{j}^{* 2}
\end{aligned}
$$

therefore, we can definitely say that $\left\langle: \hat{T}_{\mu}^{\mu}:\right\rangle \neq 0$ which means all terms of $\left\langle: \hat{T}^{\mu v}:\right\rangle$ are not zero, which shows that generators associated with spacetime symmetries which I defined earlier are broken which is what observer finds after doing Bogoliubov transformation to know about characteristics of matter in different frame.

On the other-hand if we start with the second frame from the matter action level itself without doing Bogoliubov transformation then as usual we will have Hamiltonian of following form

$$
\hat{H}=\frac{1}{2} \sum_{l} \Omega_{l}\left(\hat{b}_{l}^{\dagger} \hat{b}_{l}+\hat{b}_{l} \hat{b}_{l}^{\dagger}\right)
$$

which is invariant under $\mathrm{U}(1)$ action and therefore from partition function we can have following thermal expectation values

$$
\begin{aligned}
& \left\langle\hat{b}_{j}^{\dagger} \hat{b}_{j^{\prime}}\right\rangle_{\beta}=\frac{\delta_{i j^{\prime}}}{\mathrm{e}^{\beta \Omega_{j}}-1},\left\langle\hat{b}_{j} \hat{b}_{j^{\prime}}^{\dagger}\right\rangle_{\beta}=\frac{\delta_{i j j^{\prime}}}{1-\mathrm{e}^{-\beta \Omega_{j}}} \\
& \left\langle\hat{b}_{j} \hat{b}_{j^{\prime}}\right\rangle_{\beta}=0=\left\langle\hat{b}_{j}^{\dagger} \hat{b}_{j^{\prime}}^{\dagger}\right\rangle_{\beta}
\end{aligned}
$$

Note that in $\beta \rightarrow \infty$ limit when ground state contribution is maximum we will have

$$
\begin{aligned}
& \left\langle\hat{b}_{j}^{\dagger} \hat{b}_{j^{\prime}}\right\rangle=0, \quad\left\langle\hat{b}_{j} \hat{b}_{j^{\prime}}^{\dagger}\right\rangle=\delta_{i j^{\prime}} \\
& \left\langle\hat{b}_{j} \hat{b}_{j^{\prime}}\right\rangle=0=\left\langle\hat{b}_{j}^{\dagger} \hat{b}_{j^{\prime}}^{\dagger}\right\rangle
\end{aligned}
$$


And with the stress-energy tensor being

$$
\begin{aligned}
\hat{T}_{\mu \nu}= & \sum_{j, k}\left[\hat{b}_{j} \hat{b}_{k} \mathcal{T}_{\mu \nu}\left(g_{j}, g_{k}\right)+\hat{b}_{j}^{\dagger} \hat{b}_{k}^{\dagger} \mathcal{T}_{\mu \nu}\left(g_{j}^{*}, g_{k}^{*}\right)\right. \\
& \left.+\hat{b}_{j}^{\dagger} \hat{b}_{k} \mathcal{T}_{\mu \nu}\left(g_{j}^{*}, g_{k}\right)+\hat{b}_{j} \hat{b}_{k}^{\dagger} \mathcal{T}_{\mu \nu}\left(g_{j}, g_{k}^{*}\right)\right]
\end{aligned}
$$

where $\left\{g_{j}, g_{j}^{*}\right\}$ are two independent solutions of Klein-Gordon equation corresponding to matter field equation in target curved spacetime where we did go through Bogoliubov transformation.

With the above result it is easy to check now that in this case indeed

$$
\left\langle: \hat{T}^{\mu v}:\right\rangle=\left\langle: \hat{T}^{\mu v}:\right\rangle_{\beta \rightarrow \infty}=0
$$

which shows generators with corresponding spacetime symmetries are not broken which we should expect because classically spacetime has some Killing vector fields which defined the corresponding symmetries of the spacetime.

\section{Conclusion}

In the beginning of this article, I emphasized on the fact that in thermodynamic limit although we have 2 disjoint vector spaces but we still can do the canonical transformation. And we also restrict ourself to new Fock space because after taking infinite volume limit we can't get back to the old Fock space. In this article, I am able to show a inconsistency between Bogoliubov transformation and breaking of charges associated with classical spacetime symmetries through stress-energy tensor of matter. Although breaking of such charges in quantum field theory is not problematic but we show there are two different descriptions, one is Bogoliubov transformation through which we can find the properties of matter field in coordinate transformed spacetime (target spacetime) and other is action description of matter field imposed on the target spacetime itself from the beginning. And this also raises the question of validity of particle production phenomenon [20]-[29] which is a highly debatable matter. In the paper [30] (and others with similar approaches [31] [32] [33] [34]), the author shows that applying the rigorous algebraic approach to QFT, the derivation of the Unruh effect usually done by almost everyone is incorrect, and that the Unruh effect doesn't exist. More than that, as far as I know, the Unruh effect hasn't been yet observed therefore, in the end of the day, the Unruh effect has no any experimental observation that would point towards its correctness.

\section{Acknowledgements}

The author wants to thank Dr. Golam Mortuza Hossain for giving him opportunity to work on this topic independently. The author would also like to thank CSIR to support this work through JRF fellowship.

\section{Conflicts of Interest}

The author declares no conflicts of interest regarding the publication of this paper. 


\section{References}

[1] Mandal, S.U. (2018) Symmetry Breaking under Canonical Transformation in Real Scalar Field Theory.

[2] Perelomov, A.M. (1986) Generalized Coherent States and Their Applications of Modern Methods of Plant Analysis. Springer-Verlag, Berlin.

[3] Umezawa, H., Matsumoto, H. and Tachiki, M. (1982) Thermo Field Dynamics and Condensed States.

[4] Sato, H.-T. and Suzuki, H. (1994) On Bogoliubov Transformation of Scalar Wave Functions in de Sitter Space. Modern Physics Letters A, 9, 3673-3684. https://doi.org/10.1142/S0217732394003518

[5] Das, A. and De Benedictis, A. (2012) The General Theory of Relativity: A Mathematical Exposition. Springer-Verlag, New York.

[6] Steven, W. (1972) Gravitation and Cosmology: Principles and Applications of the General Theory of Relativity. Wiley, Hoboken.

[7] Sigbjorn, H.Y.G. (2007) Einstein's General Theory of Relativity. Springer, Berlin.

[8] Goldstone, J., Salam, A. and Weinberg, S. (1962) Broken Symmetries. Physical Review, 127, 965-970. https://doi.org/10.1103/PhysRev.127.965

[9] Schakel, A.M.J. (1998) Boulevard of Broken Symmetries.

[10] Birrell, N.D. and Davies, P.C.W. (1984) Quantum Fields in Curved Space of Cambridge Monographs on Mathematical Physics. CUP Edition, Cambridge University Press, Cambridge.

[11] Ford, L.H. (1997) Quantum Field Theory in Curved Space-Time. Particles and Fields. Proceedings, 9 th Jorge Andre Swieca Summer School, Campos do Jordao, 16-28 February 1997, 345-388.

[12] Parker, L. and Toms, D. (2009) Quantum Field Theory in Curved Spacetime: Quantized Fields and Gravity of Cambridge Monographs on Mathematical Physics. Cambridge University Press, Cambridge.

[13] Ambrus, V.E. (2014) Dirac Fermions on Rotating Space-Times. PhD Thesis, University of Sheffield.

[14] Arovas, D. (2013) Lecture Notes on Thermodynamics and Statistical Mechanics (A Work in Progress).

[15] Vilenkin, A. (1980) Quantum Field Theory at Finite Temperature in a Rotating System. Physical Review D, 21, 2260-2269. https://doi.org/10.1103/PhysRevD.21.2260

[16] Das, A. (1997) Finite Temperature Field Theory.

[17] Kapusta, J.I. (1989) Finite-Temperature Field Theory of Cambridge Monographs on Mathematical Physics. Cambridge University Press, Cambridge.

[18] Laine, M. and Vuorinen, A. (2016) Basics of Thermal Field Theory. Lecture Notes in Physics, Vol. 925, Springer, Berlin. https://doi.org/10.1007/978-3-319-31933-9

[19] Yang, Y. (2011) An Introduction to Thermal Field Theory. Imperial College, London.

[20] Ahmadzadegan, A. (2017) Probing the Unruh and Hawking Effects Using Unruh-DeWitt Detectors. PhD Thesis, U. Waterloo (Main).

[21] Birrell, N.D. and Davies, P.C.W. (1980) Massive Particle Production in Anisotropic Space-Times. Journal of Physics A: Mathematical and General, 13, 2109.

https://doi.org/10.1088/0305-4470/13/6/032 
[22] Biswas, S., Guha, J. and Sarkar, N.G. (1995) Particle Production in de Sitter Space. Classical and Quantum Gravity, 12, 1591. https://doi.org/10.1088/0264-9381/12/7/005

[23] Mo Chitre, D. and Hartle, J.B. (1977) Path-Integral Quantization and Cosmological Particle Production: An Example. Physical Review D, 16, 251. https://doi.org/10.1103/PhysRevD.16.251

[24] Crispino, L.C.B., Higuchi, A. and Matsas, G.E.A. (2008) The Unruh Effect and Its Applications. Reviews of Modern Physics, 80, 787-838. https://doi.org/10.1103/RevModPhys.80.787

[25] Davies, P.C.W. (1975) Scalar Production in Schwarzschild and Rindler Metrics. Journal of Physics A: Mathematical and General, 8, 609. https://doi.org/10.1088/0305-4470/8/4/022

[26] Degner, A. and Verch, R. (2010) Cosmological Particle Creation in States of Low Energy. Journal of Mathematical Physics, 51, 022302. https://doi.org/10.1063/1.3271106

[27] Duru, I.H. and Ünal, N. (1986) Particle Production in Expanding Universes with Path Integrals. Physical Review D, 34, 959. https://doi.org/10.1103/PhysRevD.34.959

[28] Frieman, J.A. (1989) Particle Creation in Inhomogeneous Spacetimes. Physical Review D, 39, 389-398. https://doi.org/10.1103/PhysRevD.39.389

[29] Winitzki, S. (2005) Cosmological Particle Production and the Precision of the WKB Approximation. Physical Review D, 72, Article ID: 104011. https://doi.org/10.1103/PhysRevD.72.104011

[30] Buchholz, D. and Verch, R. (2015) Macroscopic Aspects of the Unruh Effect. Classical and Quantum Gravity, 32, 245004. https://doi.org/10.1088/0264-9381/32/24/245004

[31] Blasone, M., Lambiase, G. and Luciano, G.G. (2017) Nonthermal Signature of the Unruh Effect in Field Mixing. Physical Review, D96, 025023. https://doi.org/10.1103/PhysRevD.96.025023

[32] Ford, G.W. and O’Connell, R.F. (2006) Is There Unruh Radiation? Physics Letters, A350, 17-26. https://doi.org/10.1016/j.physleta.2005.09.068

[33] Nikolic, H. (2001) Inappropriateness of the Rindler Quantization. Modern Physics Letters $A, 16,579-581$. https://doi.org/10.1142/S0217732301003681

[34] Rosu, H.C. (2001) Hawking Like Effects and Unruh Like Effects: Toward Experiments? Gravitation and Cosmology, 7, 1-17. 